\title{
Defining task-set reconfiguration: The case of reference point switching
}

\author{
DARRYl W. SChNEIDER AND GORdon D. LOGAN \\ Vanderbilt University, Nashville, Tennessee
}

\begin{abstract}
Some researchers argue that task switching involves task-set reconfiguration - that is, changing the existing task set to perform a different task. Although this idea is intriguing, it is often unclear what is reconfigured and which processes are involved in reconfiguration. We addressed these issues by defining task sets, identifying differences between them, and obtaining evidence that was diagnostic of reconfiguration. In two experiments, subjects performed relative judgment tasks that involved comparing a target with a reference point that repeated or switched across trials. The task was the same on every trial, but the task set was not, because a task-set parameter-the reference point-had to be changed. Target-reference point distance effects that changed with the reference point provided diagnostic evidence that reconfiguration occurred, and this reconfiguration led to switch costs. We discuss how our approach can be generalized to define reconfiguration more clearly in other task-switching situations.
\end{abstract}

Task switching is a hallmark of executive control, reflecting the flexibility of the cognitive system in adapting to dynamic situations. But this flexibility comes with a cost: People are slower and less accurate at switching than at repeating tasks (Monsell, 2003). Some researchers argue that this switch cost reflects task-set reconfiguration: changing the existing task set - a specific configuration of the cognitive system - to perform a different task (Meiran, 1996; Rogers \& Monsell, 1995). Although the concept of task-set reconfiguration is intriguing, it is often unclear what is reconfigured and which processes are involved in reconfiguration. Different tasks are assumed to require different task sets, but what constitutes a task set is seldom explained, the differences between task sets are rarely identified, and the distinction between tasks and task sets is hardly ever discussed. Moreover, evidence that is uniquely diagnostic of reconfiguration is rarely presented to indicate that reconfiguration actually occurred. We believe that the practice of attributing switch costs to reconfiguration in task-switching studies is problematic without a clear definition of reconfiguration and evidence that is diagnostic of reconfiguration.

These issues can be addressed by adopting clear definitions of task set and reconfiguration. Following Logan and Gordon (2001), we define a task set as a set of parameters that program task-specific processes such as perceptual encoding, memory retrieval, response selection, and response execution. Differences between task sets are differences in the parameter values for those task sets, and reconfiguration involves changing task-set parameters. Defining reconfiguration in a given situation involves identifying those parameters that differentiate task sets and linking them to processes that can change them. It is not necessary to define each task set completely if one can isolate the key difference(s) between the task sets required in an experiment.

Once reconfiguration is defined, one can seek evidence that is diagnostic of each task set and is therefore diagnostic of reconfiguration. The mere presence of a switch cost tends to be interpreted as evidence that reconfiguration has occurred, but such a cost is not diagnostic in itself because reconfiguration may not be the sole cause. ${ }^{1}$ Low error rates are also not diagnostic of reconfiguration, because reconfiguration is not a prerequisite for accurate taskswitching performance (see Schneider \& Logan, 2005). Consequently, we must look beyond switch costs and error rates to obtain evidence of reconfiguration. Ideally, this evidence should be linked to the definition of reconfiguration by finding an effect - which need not have anything to do with task switching per se - that is directly associated with a task-set parameter that is assumed to change.

For task-set reconfiguration to be a useful explanatory construct, it is important to define task sets, identify differences between them, and obtain evidence that is diagnostic of reconfiguration. Once we have a working definition of reconfiguration and evidence that it has occurred, then we can make inferences about the processes underlying reconfiguration and how they may contribute to switch costs. In the present study, we demonstrate how this can be done in the case of reference point switching.

\section{Reference Point Switching}

Consider the following relative judgments: Is the number 4 lower or higher than 2? Is 4 lower or higher than 7? Both judgments are manifestations of the same task: judging whether a target (4) is lower or higher than a refer-

D.W. Schneider, darryl.schneider@vanderbilt.edu 
ence point ( 2 or 7$)$. The task sets required for these judgments depend on identical representations, with shared parameters for the same perceptual, cognitive, and motor processes, except for a single task-set parameter: the reference point used in response selection. If the reference point switches across judgments, one could argue that the task is the same, but the task set is not because the reference point must be changed. By isolating the parameter that differentiates task sets, we can define reconfiguration in this case as switching reference points.

If reconfiguration involves switching reference points, we can find evidence that is diagnostic of reconfiguration by focusing on effects that differ with the reference point. For relative judgments, we can focus on the well-known distance effect: As the distance in psychological space between two stimuli increases, the time required to compare the stimuli decreases (Banks, 1977; Moyer \& Dumais, 1978). Distance effects implicate a reference-point-based algorithm in response selection, in which the distance between the target and the reference point is computed and used to select a response (Buckley \& Gillman, 1974; Link, 1975; Poltrock, 1989). Applied to reference point switching, distance effects should differ as a function of the reference point if different reference points are used in response selection (Dehaene, 1989; Holyoak, 1978). Changes in distance effects would provide clear evidence that reconfiguration had occurred and indicate that response selection did not depend exclusively on memory retrieval. ${ }^{2}$

Two experiments were conducted to investigate reference point switching. Each experiment involved judging whether a target was lower or higher than a reference point. In Experiment 1, subjects judged whether a target digit was lower or higher than one of two numerical reference points. In Experiment 2, they judged whether a target mark was lower or higher than one of two spatial reference points. Both experiments involved the same design and procedure, allowing us to compare data obtained for symbolic and perceptual comparisons.

On each trial, the relevant reference point was signaled by one of two cues, enabling three types of transitions across trials: cue repetitions (cue and reference point repeat), reference point repetitions (cue switches but reference point repeats), and reference point switches (cue and reference point switch). The response time (RT) difference between reference point switches and reference point repetitions reflects a switch cost, whereas the RT difference between reference point repetitions and cue repetitions reflects an encoding benefit for repeated cues. We manipulated the cue-target interval (CTI) to examine time course functions for these effects. Many researchers have found that these effects decrease as CTI increases (Logan \& Bundesen, 2003; Meiran, 1996; Schneider \& Logan, 2005), although the interaction between switch cost and CTI - which has been interpreted as evidence of advance reconfiguration (Meiran, 1996; Monsell, 2003; Monsell \& Mizon, 2006) — is not as robust as previously thought. ${ }^{3}$

If switching reference points is a form of reconfiguration, we should obtain different distance effects that are diagnostic of this reconfiguration. If reconfiguration oc- curs, we should also obtain a switch cost. Whether or not switch cost will decrease with CTI is unclear (see note 3), but the interaction is secondary to simply observing a switch cost. If cue encoding is susceptible to priming, we should obtain a repeated-cue encoding benefit. This effect should decrease with CTI because a longer CTI provides more time for cue encoding to finish before target onset, so differences in cue encoding time will be smaller at longer CTIs (Logan \& Bundesen, 2003).

Finally, half of the targets were always mapped to the same response (consistent targets), and half were mapped to different responses depending on the reference point (varied targets). Based on the automaticity literature (Logan, 1988), we should obtain a mapping effect: faster RTs for consistent targets than for varied targets, because response selection for consistent targets could involve memory retrieval of past responses. This effect would not depend on reconfiguration and seems inconsistent with the proposal that response selection is algorithmic, raising an interesting question: If memory retrieval is used for consistent targets, will different distance effects and switch costs only be observed for varied targets?

\section{METHOD}

\section{Subjects}

Seventy-two individuals ( 36 per experiment) from Vanderbilt University participated for course credit or monetary compensation.

\section{Apparatus and Stimuli}

Both experiments used E-Prime software (Psychology Software Tools, Pittsburgh, PA) operating on computers that registered input from keyboards and displayed the output on monitors. Each experiment involved judging whether a target was lower or higher than a reference point indicated by a cue. Experiment 1 involved numerical judgments: the targets were $0,1,3,4,5,6,8$, and 9 , and the reference points were 2 and 7 (each digit was displayed in white and measured $9 \times 14 \mathrm{~mm}$ ). Experiment 2 involved spatial judgments: The targets were eight white vertical marks, and the reference points were two gray vertical marks (each mark measured $2 \times 13 \mathrm{~mm}$ ) on a gray horizontal line $(290 \times 2 \mathrm{~mm}$; adjacent marks were separated by $30 \mathrm{~mm}$ ). The correspondence between targets and reference points across experiments is depicted in Figure 1; for convenience, the spatial positions of the marks in Experiment 2 will be referenced with the corresponding digits from Experiment 1. Targets were divided into two categories based on their mapping to responses: $0,1,8$, and 9 were consistent targets because they always required the same response, regardless of the reference point; $3,4,5$, and 6 were varied targets because they required different responses with different reference points. Each reference point was associated with two cues from a set of four shapes: circle, diamond, triangle, and square. Each shape was displayed as a white outline and measured $29 \times 29 \mathrm{~mm}$. The assignment of cues to reference points was counterbalanced across subjects. All stimuli were displayed on a black background and viewed at a distance of about $60 \mathrm{~cm}$.

\section{Procedure}

Subjects completed the experiments in private testing rooms. Instructions concerning the task, reference points, cues, targets, trial format, and response-key mappings were presented onscreen and explained by the experimenter. For Experiment 2, the subjects were told that any target appearing to the left of a reference point was lower and any to the right was higher.

The experiment was divided into blocks of 80 trials. Each trial had the following format: First, a cue was displayed in the center of the 


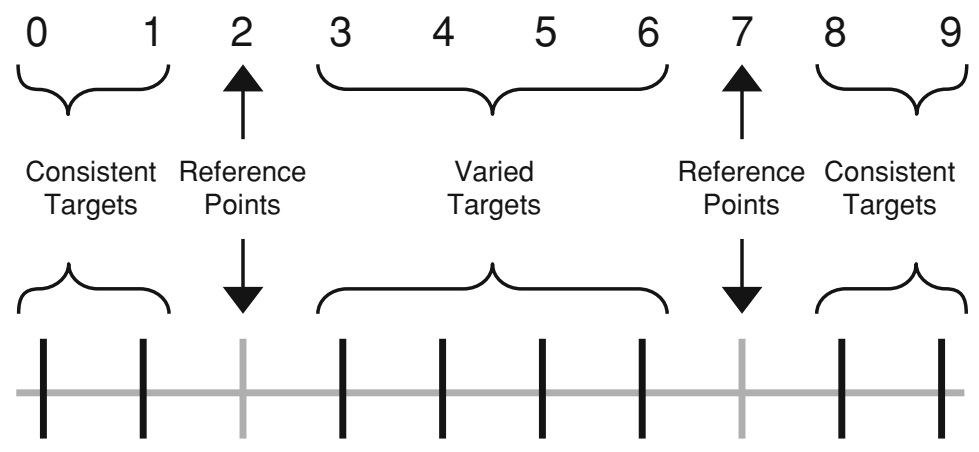

Figure 1. Illustration of the corresponding targets (consistent and varied) and reference points for the numerical and spatial judgments in Experiments 1 and 2 , respectively. Target marks are depicted in black for visibility.

screen. After a variable CTI $(0,100,200,400$, or $800 \mathrm{msec})$, a target appeared $25 \mathrm{~mm}$ below the cue. In Experiment 1, the target appeared directly below the cue; in Experiment 2, the target appeared in the appropriate spatial position on a horizontal line that was below the cue. The cue and the target remained onscreen until the subject responded by pressing the $F$ key with the left index finger for lower or the $J$ key with the right index finger for higher. After a response, the cue and the target were erased, and the next trial commenced after $500 \mathrm{msec}$. In Experiment 2, the horizontal line with both reference point marks remained visible throughout the trial. The response and RT were recorded for every trial.

The cue, target, and CTI on each trial were randomly selected from a 4 (cue) $\times 8$ (target) $\times 5(\mathrm{CTI})$ block design. Each experiment included 1 block of 40 practice trials, followed by blocks of 80 experimental trials (10 blocks in Experiment 1; 12 in Experiment 2). Blocks were separated by rest periods, and every 2 blocks represented one replication of the block design.

\section{RESULTS}

The practice block, the first trial of each experimental block, and trials with RTs exceeding 3,000 msec (2.6\% and $1.4 \%$ of trials in Experiments 1 and 2, respectively) were excluded from analysis. The transition for each trial was classified as a reference point switch, reference point repetition, or cue repetition on the basis of the relationship between trials $n$ and $n-1$. Target mapping for each trial was classified as consistent or varied according to Figure 1 . We conducted two sets of analyses. The first set focused on distance and mapping effects, to determine whether reconfiguration occurred. The second set focused on transition, mapping, and CTI effects, to assess the effects of reconfiguration.

\section{Distance and Mapping Effects}

Mean RT and error rate were calculated for each combination of reference point and target; these data are provided in Table 1 and were subjected to separate 2 (reference point $) \times 8$ (target) repeated measures ANOVAs for each experiment (summarized in Table 2). The error rate data were consistent with the RT data; therefore, we focused on the RT analysis of correct trials.

There was no main effect of reference point, but there was a main effect of target and a reference point $\times$ target interaction in each experiment. The interactions could only arise if different reference points were used across trials to perform the judgment. Planned contrasts were conducted using the error terms from the interactions to assess the significance of specific interactions that index changes in distance effects separately for varied and consistent targets (see Figure 2).

For varied targets, a contrast was conducted using the weights $+3,+1,-1$, and -3 for the targets $3,4,5$, and 6 with reference point 2 and the weights $-3,-1,+1$, and +3 , respectively, for those targets with reference point 7 ; this contrast represents a symmetrical X-shaped interaction, in which RT decreases linearly as the distance between the

Table 1

Mean Response Time (RT, in Milliseconds) and Error Rate (ER, As a Percentage of Incorrect Responses), With Standard Errors, Across Subjects As a Function of Reference Point (RP) and Target in Experiments 1 and 2

\begin{tabular}{|c|c|c|c|c|c|c|c|c|c|c|c|c|c|c|c|c|}
\hline \multirow[b]{3}{*}{ Target } & \multicolumn{8}{|c|}{ Experiment 1} & \multicolumn{8}{|c|}{ Experiment 2} \\
\hline & \multicolumn{4}{|c|}{ RP 2} & \multicolumn{4}{|c|}{ RP 7} & \multicolumn{4}{|c|}{ RP 2} & \multicolumn{4}{|c|}{ RP 7} \\
\hline & RT & $S E$ & ER & $S E$ & RT & $S E$ & ER & $S E$ & RT & $S E$ & ER & $S E$ & RT & $S E$ & ER & $S E$ \\
\hline 0 & 795 & 24 & 2.9 & 0.6 & 774 & 25 & 0.7 & 0.3 & 701 & 23 & 2.2 & 0.5 & 657 & 21 & 0.3 & 0.1 \\
\hline 1 & 879 & 27 & 5.3 & 1.0 & 827 & 31 & 0.6 & 0.2 & 738 & 25 & 2.4 & 0.5 & 684 & 24 & 0.3 & 0.1 \\
\hline 3 & 1,002 & 35 & 6.4 & 1.0 & 969 & 34 & 2.6 & 0.5 & 806 & 33 & 5.4 & 0.8 & 805 & 31 & 4.6 & 0.6 \\
\hline 4 & 985 & 30 & 3.6 & 0.6 & 970 & 33 & 1.8 & 0.4 & 783 & 32 & 4.8 & 0.7 & 778 & 29 & 3.3 & 0.5 \\
\hline 5 & 942 & 33 & 3.3 & 0.5 & 984 & 33 & 3.1 & 0.6 & 760 & 31 & 3.0 & 0.5 & 790 & 29 & 4.5 & 0.7 \\
\hline 6 & 959 & 36 & 2.9 & 0.5 & 1,028 & 34 & 4.4 & 0.8 & 792 & 31 & 4.4 & 0.6 & 832 & 34 & 5.6 & 0.6 \\
\hline 8 & 814 & 28 & 0.5 & 0.2 & 904 & 31 & 6.0 & 1.1 & 699 & 25 & 0.4 & 0.2 & 739 & 28 & 1.8 & 0.4 \\
\hline 9 & 776 & 28 & 0.4 & 0.2 & 831 & 28 & 5.4 & 1.0 & 661 & 21 & 0.3 & 0.1 & 702 & 25 & 2.5 & 0.4 \\
\hline
\end{tabular}


Table 2

Summary Table for the ANOVAs Conducted on Mean Response Time and Error Rate As a Function of Reference Point and Target in Experiments 1 and 2

\begin{tabular}{|c|c|c|c|c|c|c|c|c|c|c|c|c|c|}
\hline \multirow[b]{3}{*}{ Effect } & \multirow[b]{3}{*}{$d f$} & \multicolumn{6}{|c|}{ Experiment 1} & \multicolumn{6}{|c|}{ Experiment 2} \\
\hline & & \multicolumn{3}{|c|}{ Response Time } & \multicolumn{3}{|c|}{ Error Rate } & \multicolumn{3}{|c|}{ Response Time } & \multicolumn{3}{|c|}{ Error Rate } \\
\hline & & $F$ & $M S_{\mathrm{e}}$ & $\eta_{\mathrm{p}}^{2}$ & $F$ & $M S_{\mathrm{e}}$ & $\overline{\eta_{\mathrm{p}}^{2}}$ & $F$ & $M S_{\mathrm{e}}$ & $\overline{\eta_{\mathrm{p}}^{2}}$ & $F$ & $M S_{\mathrm{e}}$ & $\overline{\eta_{\mathrm{p}}^{2}}$ \\
\hline Reference point (R) & 1,35 & 2.57 & $15,768.35$ & .07 & 0.13 & 8.32 & .00 & 0.86 & $5,780.62$ & .02 & 0.00 & 8.18 & .00 \\
\hline Target $(\mathrm{T})$ & 7,245 & $45.09^{* *}$ & $11,969.95$ & .56 & $5.73^{* *}$ & 7.58 & .14 & $20.80^{* *}$ & $10,121.13$ & .37 & $31.57^{* *}$ & 6.91 & .47 \\
\hline $\mathrm{R} \times \mathrm{T}$ & 7,245 & $9.65^{* *}$ & $5,225.81$ & .22 & $18.82^{* *}$ & 13.94 & .35 & $7.93^{* *}$ & $3,364.41$ & .19 & $7.52^{* *}$ & 7.30 & .18 \\
\hline
\end{tabular}

target and the reference point increases. This contrast was significant in Experiment $1[F(1,245)=22.61, p<.01]$ and Experiment $2[F(1,245)=6.70, p<.05]$, although the crossover is asymmetric in Experiment 2 (see Figure 2).

For consistent targets, a contrast was conducted using the weights $+1,+2,-1$, and -2 for the targets $0,1,8$, and 9 with reference point 2 and the weights $-2,-1$, +2 , and +1 , respectively, for those targets with refer-
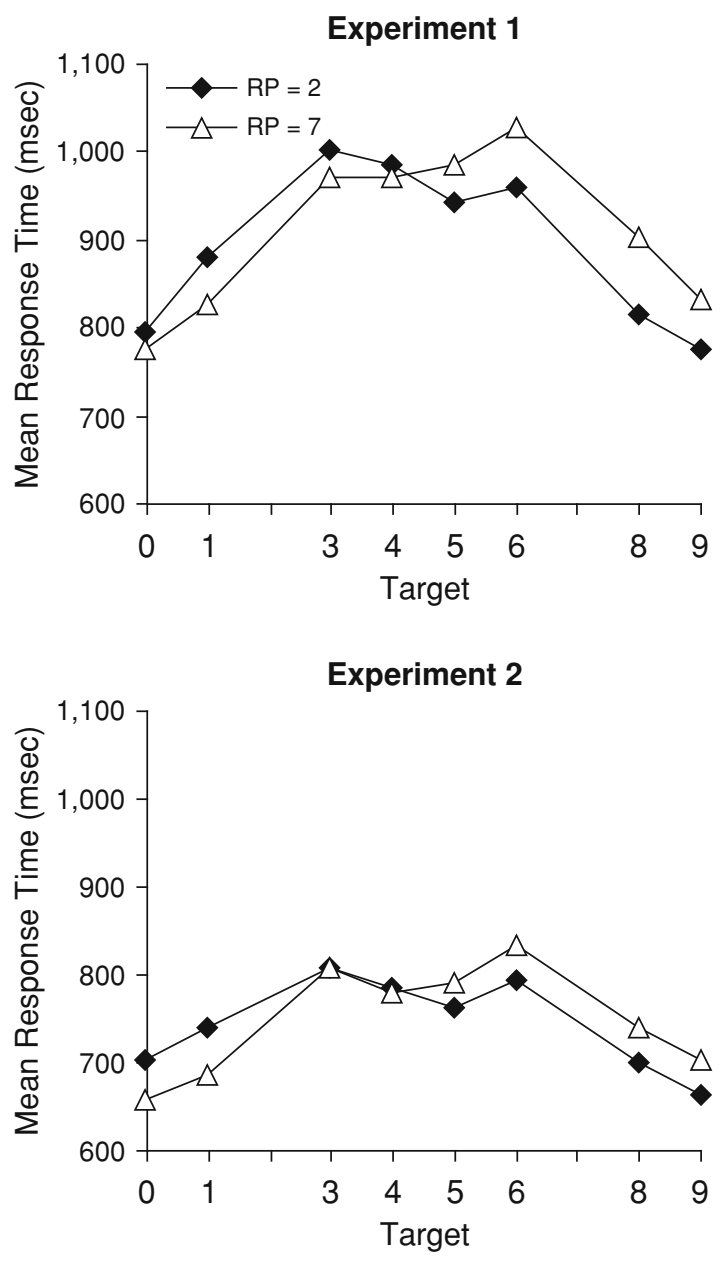

Figure 2. Mean response time with each reference point (RP) as a function of target in Experiments 1 and 2 (top and bottom panels, respectively). The spatial positions of the targets in Experiment 2 have been labeled with the corresponding digits from Experiment 1 (see Figure 1). ence point 7; this contrast represents an interaction that resembles a crossed pair of upside-down hockey sticks, in which RT decreases linearly as the distance between the target and the reference point increases, but targets 0 and 1 yield slower responses with reference point 2 than with reference point 7 , and targets 8 and 9 yield slower responses with reference point 7 than with reference point 2 (see Figure 2). This contrast was also significant in Experiment $1[F(1,245)=69.40, p<.01]$ and Experiment 2 $[F(1,245)=61.23, p<.01]$. The significant contrasts indicate that response selection involved a reference-pointbased algorithm and that this algorithm was reconfigured when switching reference points - even for consistent targets.

\section{Transition, Mapping, and CTI Effects}

Mean RT and error rate were calculated for each combination of transition, mapping, and CTI; these data are provided in Table 3 and were subjected to separate 3 (transition) $\times 2$ (mapping) $\times 5(\mathrm{CTI})$ repeated measures ANOVAs for each experiment (summarized in Table 4). The error rate data were consistent with the RT data; therefore, we focused on the RT analysis of correct trials.

There was a main effect of transition in each experiment, and planned contrasts revealed significant switch costs (47 and $38 \mathrm{msec}$ in Experiments 1 and 2, respectively; both $p$ s <.01) and repeated-cue encoding benefits (116 and $80 \mathrm{msec}$ in Experiments 1 and 2, respectively; both $p$ s <.01). The time course functions plotted in Figure 3 reveal the main effect of CTI that is usually obtained in cuing procedures (Logan \& Bundesen, 2003). As CTI increased from 0 to $800 \mathrm{msec}$, mean RT decreased monotonically from 1,034 to $736 \mathrm{msec}$ in Experiment 1 and from 844 to $641 \mathrm{msec}$ in Experiment 2.

Changes in switch cost and the repeated-cue encoding benefit with CTI were reflected in the transition $\times$ CTI interaction (see Figure 3), which was marginally significant in Experiment $1(p=.07)$ but highly significant in Experiment $2(p<.01$; see Table 4$)$. Simple interaction analyses were conducted to determine which effect (switch cost, repeated-cue encoding benefit, or both) varied with CTI. In Experiment 1, switch cost was invariant across CTI [41 and $68 \mathrm{msec}$ at CTIs of 0 and $800 \mathrm{msec}$, respectively; $F(4,280)=1.44, p=.22]$, whereas the repeated-cue encoding benefit decreased across CTI [114 and $73 \mathrm{msec}$ at CTIs of 0 and $800 \mathrm{msec}$, respectively; $F(4,280)=2.60, p<.05]$. In Experiment 2, switch cost was invariant across CTI ( 31 and $36 \mathrm{msec}$ at CTIs of 0 and 


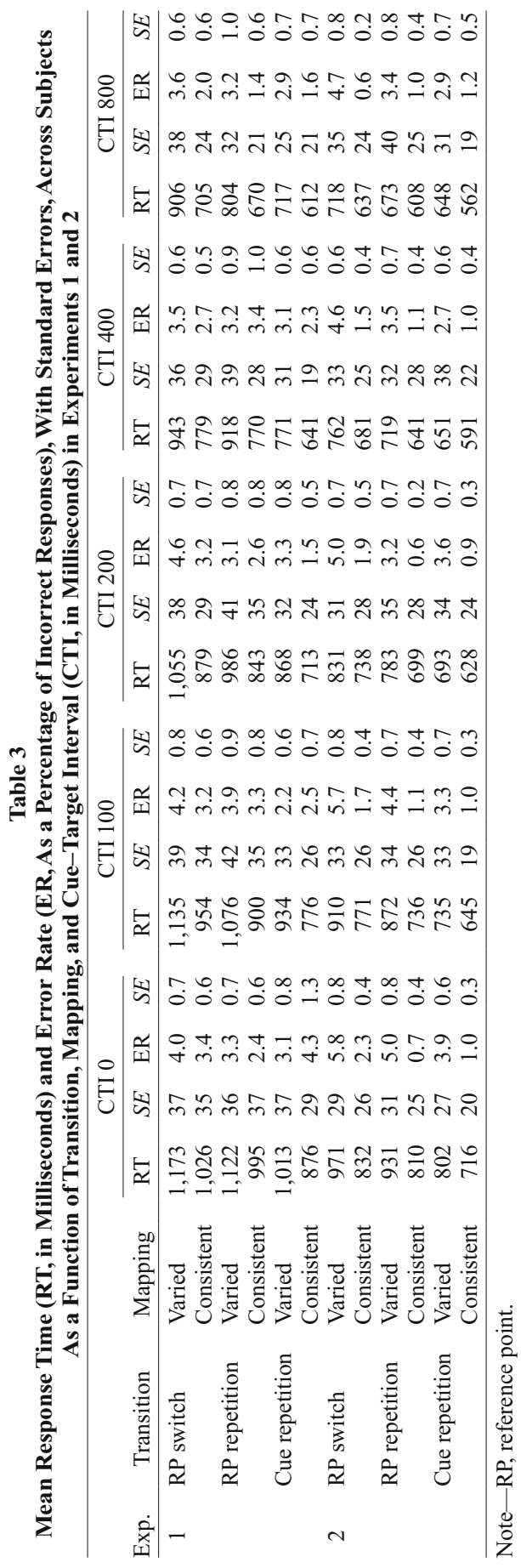

800 msec, respectively; $F<1$ ), whereas the repeated-cue encoding benefit decreased across CTI [111 and $36 \mathrm{msec}$ at CTIs of 0 and $800 \mathrm{msec}$, respectively; $F(4,280)=8.66$, $p<.01]$. The lack of an interaction between switch cost and CTI is not new (see note 3 ).

The main effect of mapping in each experiment (see Table 4) is evident in Figure 3 (compare the left and right columns, representing varied and consistent targets, respectively). Mean RT was slower for varied targets than for consistent targets by 152 and 94 msec in Experiments 1 and 2, respectively, suggesting that subjects benefited from memory retrieval with consistent targets or suffered interference from memory retrieval with varied targets. Changes in switch cost as a function of mapping are of interest because if subjects responded by memory retrieval for most of the consistent targets, switch cost should be smaller for consistent targets than for varied targets. Simple interaction analyses were conducted for the small but significant transition $\times$ mapping interactions (see Table 4). In Experiment 1, switch cost was $61 \mathrm{msec}$ for varied targets and $33 \mathrm{msec}$ for consistent targets, a marginally significant difference $[F(1,70)=3.67, p=$ .06]; the repeated-cue encoding benefit was $120 \mathrm{msec}$ for varied targets and $112 \mathrm{msec}$ for consistent targets, a nonsignificant difference $(F<1)$. In Experiment 2, switch cost was $42 \mathrm{msec}$ for varied targets and $33 \mathrm{msec}$ for consistent targets, a nonsignificant difference $(F<1)$; the repeated-cue encoding benefit was $90 \mathrm{msec}$ for varied targets and $71 \mathrm{msec}$ for consistent targets, a marginally significant difference $[F(1,70)=3.08, p=.08]$. The presence of a mapping effect, coupled with distance effects and switch costs for consistent targets, suggests that response selection involved both memory retrieval and algorithmic processing.

\section{DISCUSSION}

Both experiments revealed a number of critical effects. There were reference point $\times$ target interactions for both varied and consistent targets, reflecting distance effects that indicate the use of different reference points across trials. These effects are diagnostic of reconfiguration: The task set for performing the judgment was reconfigured by changing the reference point. This reconfiguration led to a switch cost: RT was slower for reference point switches than for reference point repetitions, an effect that did not vary with CTI. Using two cues per reference point, we separated switch cost from the repeated-cue encoding benefit: RT was faster for cue repetitions than for reference point repetitions, an effect that decreased with CTI. The repeated-cue encoding benefit suggests priming of cue encoding, as observed in previous studies (Logan \& Bundesen, 2003; Schneider \& Logan, 2005). Finally, there was a mapping effect: Varied targets yielded slower responses than did consistent targets, but switch costs were present for both target types, suggesting that memory retrieval and algorithmic processing both contributed to response selection, perhaps racing to produce a response on each trial (Logan, 1988). 
Table 4

Summary Table for the ANOVAs Conducted on Mean Response Time and Error Rate As a Function of Transition, Mapping, and Cue-Target Interval (CTI) in Experiments 1 and 2

\begin{tabular}{|c|c|c|c|c|c|c|c|c|c|c|c|c|c|}
\hline \multirow[b]{3}{*}{ Effect } & \multirow[b]{3}{*}{$d f$} & \multicolumn{6}{|c|}{ Experiment 1} & \multicolumn{6}{|c|}{ Experiment 2} \\
\hline & & \multicolumn{3}{|c|}{ Response Time } & \multicolumn{3}{|c|}{ Error Rate } & \multicolumn{3}{|c|}{ Response Time } & \multicolumn{3}{|c|}{ Error Rate } \\
\hline & & $F$ & $M S_{\mathrm{e}}$ & $\eta_{\mathrm{p}}^{2}$ & $F$ & $M S_{\mathrm{e}}$ & $\eta_{\mathrm{p}}^{2}$ & $F$ & $M S_{\mathrm{e}}$ & $\eta_{\mathrm{p}}^{2}$ & $F$ & $M S_{\mathrm{e}}$ & $\eta_{\mathrm{p}}^{2}$ \\
\hline Transition (T) & 2,70 & $98.39^{* *}$ & $25,887.26$ & .74 & 2.94 & 18.66 & .08 & $58.07^{* *}$ & $22,419.76$ & .62 & $11.90^{* *}$ & 12.77 & .25 \\
\hline Mapping (M) & 1,35 & $63.47^{* *}$ & $98,577.48$ & .65 & $5.12^{*}$ & 30.03 & .13 & $23.60^{* *}$ & $100,501.70$ & .40 & $64.13^{* *}$ & 36.62 & .65 \\
\hline CTI (C) & 4,140 & $167.01^{* *}$ & $18,459.52$ & .83 & 1.78 & 15.20 & .05 & $163.34^{* *}$ & $8,666.87$ & .82 & $3.04^{*}$ & 8.53 & .08 \\
\hline $\mathrm{T} \times \mathrm{M}$ & 2,70 & $3.41^{*}$ & $9,851.60$ & .09 & 0.52 & 15.28 & .01 & $3.53^{*}$ & $5,522.31$ & .09 & $3.94^{*}$ & 10.06 & .10 \\
\hline $\mathrm{T} \times \mathrm{C}$ & 8,280 & 1.83 & $9,380.48$ & .05 & 1.16 & 13.70 & .03 & $5.53^{* *}$ & $4,751.63$ & .14 & 0.73 & 8.68 & .02 \\
\hline $\mathrm{M} \times \mathrm{C}$ & 4,140 & 0.93 & $10,108.11$ & .03 & 1.40 & 14.85 & .04 & $6.81^{* *}$ & $4,159.86$ & .16 & 1.09 & 9.81 & .03 \\
\hline $\mathrm{T} \times \mathrm{M} \times \mathrm{C}$ & 8,280 & 0.76 & $8,115.45$ & .02 & 0.77 & 12.16 & .02 & 0.77 & $4,852.70$ & .02 & 0.62 & 7.98 & .02 \\
\hline
\end{tabular}

${ }^{*} p<.05 . \quad{ }^{* *} p<.01$.

Collectively, these effects allow us to interpret reference point switching as a well-defined form of reconfiguration. By defining reconfiguration and finding evidence that is diagnostic of reconfiguration, we are now in a better position to make inferences about the processes underlying reconfiguration and how they may contribute to switch costs.

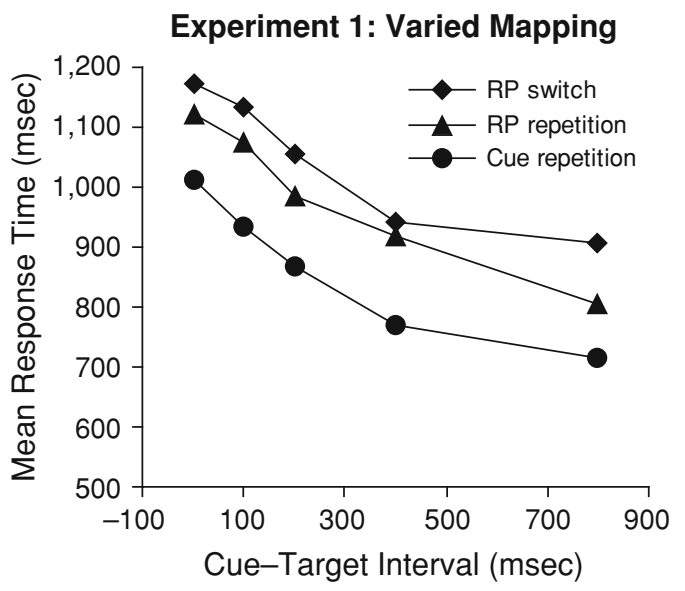

Experiment 2: Varied Mapping

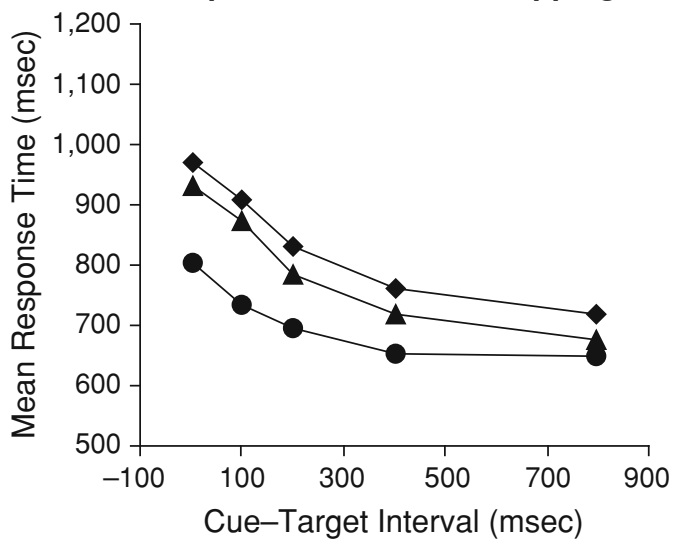

Reference Point Switching as Attention Shifting

In our experiments, subjects had to attend to the cue and the target to obtain the necessary information to perform the task. We suggest that switching reference points is analogous to shifting attention along the dimension associated with the reference points and targets. This interpretation is simplest when applied to Experiment 2, in which attention
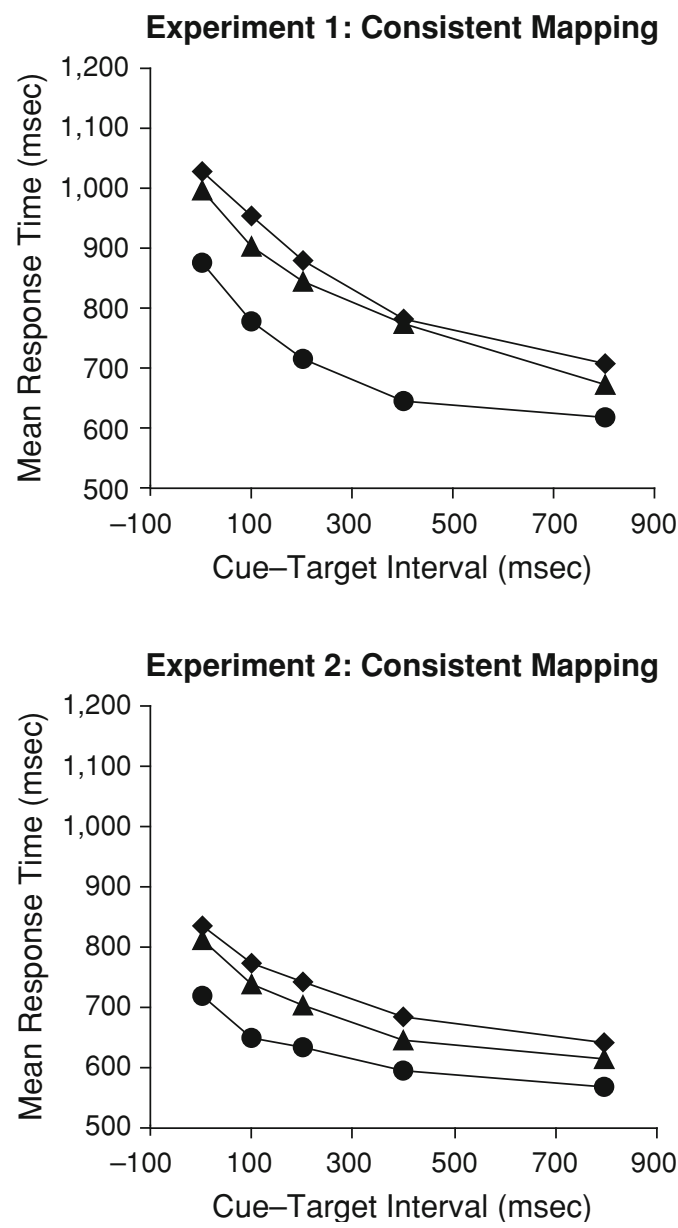

Figure 3. Mean response time for each transition, as a function of cue-target interval for varied and consistent targets (left and right columns, respectively), in Experiments 1 and 2 (top and bottom rows, respectively). RP, reference point. 
could be focused on the reference point mark indicated by the cue. When switching reference points, attention could be shifted along the horizontal line from one reference point to the other. From this perspective, the task-set parameter that was changed was an attentional control setting, which is clearly defined in computational models of executive control (Logan \& Gordon, 2001) and could be implemented in extensions to computational models of relative judgment (Buckley \& Gillman, 1974; Link, 1975; Poltrock, 1989).

The same interpretation can be applied to Experiment 1, except that assumptions must be made about the internal representation of the reference points and targets. We assume that our numerical stimuli were represented spatially along a mental number line, consistent with research on numerical cognition (Fias \& Fischer, 2005). As depicted in Figure 1, the mental number line consists of numbers arrayed from left to right in order of increasing magnitude. The prevalence of the mental number line in numerical cognition was part of our motivation for conducting experiments with symbolic and perceptual comparisons. If both comparisons require access to an internal or external spatial representation of the stimuli, similar data should be produced. It is evident from Figures 2 and 3 that the critical effects were comparable across experiments, supporting our interpretation and Moyer and Dumais's (1978) suggestion that symbolic and perceptual comparisons act on similar representations.

If the numerical stimuli were represented spatially in Experiment 1, we can then interpret a switch in reference points as a shift in attention along the mental number line. This interpretation for numerical stimuli is identical to that for spatial stimuli, with the distinction that the former involves an internal and the latter an external focus of attention. The properties of shifting attention may be similar in both cases. Consequently, switching reference points may involve changing a task-set parameter that shifts attention, linking reconfiguration to a well-defined process that could be instantiated in computational models (Buckley \& Gillman, 1974; Link, 1975; Poltrock, 1989).

Switch costs may arise from the act of reconfiguration or emerge as a consequence. The former view implies that switch costs are indices of the time it takes to shift attention, an interpretation consistent with some extant research (Logan, 2005). The latter implies that switch costs arise from altered postreconfiguration processing, an interpretation consistent with the idea that task performance is susceptible to priming from past stimuli, such as the previously attended reference point (see note 1). Although we cannot distinguish between these possibilities, the overarching point is that by defining reconfiguration and finding evidence that it occurred, we are now in a position to evaluate these possibilities in future research.

\section{Beyond Reference Point Switching}

The basic strategy of defining task sets, identifying differences between them, and obtaining evidence that is diagnostic of reconfiguration was demonstrated here with reference point switching, but it can be easily generalized to more typical task-switching paradigms. We chose to use a single task with two task sets to make a clear distinction between tasks and task sets, but using multiple tasks with multiple task sets is simply an extension of our strategy. All that would be required is a careful analysis of the differences between task sets and a search for task-specific effects that implicate different task sets in performance (rather than merely a single task set based on memory retrieval) and that can be derived from computational models of task-specific processes. If this can be accomplished, then one is in a better position to make inferences about how reconfiguration leads to switch costs, allowing for better insight into the "sort of mental "gear changing" (Monsell, 2003, p. 135) that is thought to underlie task switching and executive control.

\section{AUTHOR NOTE}

This research was supported by Grants BCS 0133202 and BCS 0446806 from the National Science Foundation to G.D.L. We thank Eric Ruthruff, Bruce Milliken, and an anonymous reviewer for comments on a previous draft of this article. Correspondence concerning this article should be addressed to D. W. Schneider, Department of Psychology, Vanderbilt University, Nashville, TN 37203 (e-mail: darryl.schneider@ vanderbilt.edu).

\section{REFERENCES}

Allport, A., \& Wylie, G. (2000). Task switching, stimulus-response bindings, and negative priming. In S. Monsell \& J. Driver (Eds.), Control of cognitive processes: Attention and performance XVIII (pp. 3570). Cambridge, MA: MIT Press.

Arrington, C. M., \& Logan, G. D. (2004). Episodic and semantic components of the compound-stimulus strategy in the explicit taskcuing procedure. Memory \& Cognition, 32, 965-978.

BANKS, W. P. (1977). Encoding and processing of symbolic information in comparative judgments. In G. H. Bower (Ed.), The psychology of learning and motivation: Advances in research and theory (Vol. 11, pp. 101-159). New York: Academic Press.

Buckley, P. B., \& Gillman, C. B. (1974). Comparisons of digits and dot patterns. Journal of Experimental Psychology, 103, 1131-1136.

Dehaene, S. (1989). The psychophysics of numerical comparison: A reexamination of apparently incompatible data. Perception \& Psychophysics, 45, 557-566.

FIAS, W., \& Fischer, M. H. (2005). Spatial representation of numbers. In J. I. D. Campbell (Ed.), Handbook of mathematical cognition (pp. 4354). New York: Psychology Press.

HOLYOAK, K. J. (1978). Comparative judgments with numerical reference points. Cognitive Psychology, 10, 203-243.

LINK, S. W. (1975). The relative judgment theory of two choice response time. Journal of Mathematical Psychology, 12, 114-135.

Logan, G. D. (1988). Toward an instance theory of automatization. Psychological Review, 95, 492-527.

Logan, G. D. (2005). The time it takes to switch attention. Psychonomic Bulletin \& Review, 12, 647-653.

Logan, G. D., \& Bundesen, C. (2003). Clever homunculus: Is there an endogenous act of control in the explicit task-cuing procedure? Journal of Experimental Psychology: Human Perception \& Performance, 29, 575-599.

Logan, G. D., \& Gordon, R. D. (2001). Executive control of visual attention in dual-task situations. Psychological Review, 108, 393-434.

MaYr, U., \& KLIEGL, R. (2003). Differential effects of cue changes and task changes on task-set selection costs. Journal of Experimental Psychology: Learning, Memory, \& Cognition, 29, 362-372.

Meiran, N. (1996). Reconfiguration of processing mode prior to task performance. Journal of Experimental Psychology: Learning, Memory, \& Cognition, 22, 1423-1442.

Monsell, S. (2003). Task switching. Trends in Cognitive Sciences, 7 , 134-140.

Monsell, S., \& Mizon, G. A. (2006). Can the task-cuing paradigm mea- 
sure an endogenous task-set reconfiguration process? Journal of Experimental Psychology: Human Perception \& Performance, 32, 493-516.

Moyer, R. S., \& DumaIs, S. T. (1978). Mental comparison. In G. H. Bower (Ed.), The psychology of learning and motivation: Advances in research and theory (Vol. 12, pp. 117-155). New York: Academic Press.

Poltrock, S. E. (1989). A random walk model of digit comparison. Journal of Mathematical Psychology, 33, 131-162.

Rogers, R. D., \& Monsell, S. (1995). Costs of a predictable switch between simple cognitive tasks. Journal of Experimental Psychology: General, 124, 207-231.

Schneider, D. W., \& Logan, G. D. (2005). Modeling task switching without switching tasks: A short-term priming account of explicitly cued performance. Journal of Experimental Psychology: General, 134, 343-367.

WaszaK, F., Hommel, B., \& Allport, A. (2003). Task-switching and long-term priming: Role of episodic stimulus-task bindings in taskshift costs. Cognitive Psychology, 46, 361-413.

\section{NOTES}

1. The interpretation of switch costs as evidence for reconfiguration seems to be based on the following argument: "If reconfiguration occurs, then there will be a switch cost. And there is a switch cost, therefore reconfiguration occurred." This argument is an example of the logical fallacy of affirming the consequent - assuming that the converse of a statement is true when it need not be. Applied to task switching, understanding the fallacy involves recognizing that switch costs may emerge from alternative sources, such as positive and negative priming from stimuli, cues, tasks, and responses in memory (see, e.g., Allport \& Wylie, 2000; Logan \& Bundesen, 2003; Schneider \& Logan, 2005;
Waszak, Hommel, \& Allport, 2003). Schneider and Logan even argued that switch costs may arise solely from priming effects, in the absence of reconfiguration.

2. Ruling out the idea that response selection occurs exclusively by memory retrieval is important because Schneider and Logan (2005) argued that nominally different tasks could be performed by compoundcue retrieval, in which a task cue (or reference point in the present context) and a target are used as joint retrieval cues to select a response from memory. Compound-cue retrieval requires only a single task set, making reconfiguration unnecessary. It is unclear how distance effects in reference point switching could arise solely from memory retrieval; therefore, such effects suggest the existence of an algorithmic comparison process in the task set that can be reconfigured.

3. Many cued task-switching studies involve only one cue per task, enabling only cue repetitions (i.e., cue and task repeat) and task switches (i.e., cue and task switch), with "switch cost" defined as the difference between them. Although this switch cost tends to decrease as CTI increases, cue repetitions are confounded with task repetitions, so that the interaction may reflect a decrease in the repeated-cue encoding benefit rather than the "true" switch cost. Indeed, in recent task-switching studies involving two cues per task - a paradigm that allows one to separate the repeated-cue encoding benefit from the true switch cost-an interaction between true switch cost and CTI has not been consistently observed (see, e.g., Arrington \& Logan, 2004; Mayr \& Kliegl, 2003; Monsell \& Mizon, 2006).

(Manuscript received November 28, 2005; revision accepted for publication April 25, 2006.) 